Classification

Physics Abstracts

$82.80-01.65-06.50$

\title{
Microanalysis and on-line computers
}

\author{
Kurt F.J. Heinrich \\ 804 Blossom Dr., Rockville, MD, U.S.A. \\ (Received March 13, 1992; accepted June 01, 1992)
}

\begin{abstract}
The use of on-line computers in electron probe microanalysis permits obtaining results of complex correction calculations almost simultaneously with the measurement. Numerical operations can be used to advantage, replacing formal integration, and simplifications in the procedure which previously were unavoidable are no longer necessary.
\end{abstract}

In his doctoral thesis [1] and subsequent publications [2, 3], R. Castaing has provided us with a complete procedure of quantitative analysis, the principles of which still stand today. Yet, the practice of data reduction, its speed and accuracy, have changed substantially. The reasons for the improvements achieved are several:

1. In deriving procedures for the calculations of $x$-ray generation and absorption, Castaing had to resort to the physics of the thirties. Several proposed models for the behavior of electrons in matter were gross approximations which had not, of course, been deviced originally for the application in microanalysis.

2. Important physical parameters, such as the $x$-ray mass absorption coefficients, ionization cross-sections and expressions for stopping power (mean ionization potentials, or "J-factors") were not adequately known.

3. The reproducibility and stability of electronic and mechanical parts of the instrument were inferior to those obtainable at present.

4. Observable photon energies did not include those below one keV; energy-dispersive solidstate detectors were not available.

5. On line computers did not exist.

The last point was crucial in the choice of procedures for data reduction. Any practical study of a specimen must perform many measurements at various microscopic sites on the specimen; the composition of the specimen at one single point is hardly ever of any practical interest, and the results of previous measurements frequently determine the subsequent analytical steps. Hence, on-line analysis is an almost indispensable requisite, but the time involved in hand-calculating the estimates of composition was a serious limitation.

As shown in the thesis of Castaing, two aspects of the production of $x$-rays are of fundamental importance in quantitative microanalysis: the generation of characteristic $x$-ray photons per electron, and the depth distribution within the specimen of the generation of primary x-ray photons. The first involves what became known as the "atomic number effect"; the second governs 
the spatial resolution of the analysis as well as the attenuation of the x-rays on their path within the specimen, described in the "absorption correction". For the "atomic number effect", Castaing was forced to postulate very simple models of electron deceleration, which would lead to manageable approaches of calculation with the means available at that time $[1,2]$. One aspect which later showed to be significant, the loss of potential $x$-ray generation by backscattering energetic electrons, or "backscatter correction", was not taken explicity into account until it was recognized by Castaing in 1960 [3]. Duncumb and Reed [4] calculated the backscatter loss on the basis of Monte-Carlo calculations of Bishop for three elements, and provided a polynomial expression for this effect.

J. Philibert greatly improved the situation by providing an algebraic model for the absorption of primary x-rays within the target, in 1963 [5]. In the derivation of his model, he was forced to adopt a series of drastic simplifications in order to obtain an expression that could be used in practice. As a consequence, his final formula for the absorption of primary radiation within the specimen corresponds to a distribution of $x$-ray production with a value of zero at zero depth: no $x$-rays would be generated at the surface. This anomaly, forced upon the analyst by expedience, was not part of Philibert's original assumptions. At a time when the $x$-rays of elements of atomic number below ten would not be measured with the available spectrometers, and when there was no demand as yet for the analysis of layers and coatings within the depth of electron penetration, this simplication could be considered acceptable. For years to come, the main research effort was directed towards obtaining absorption formulae of simple structure that would provide reasonably low errors in the analysis of known specimens. Little attention was given to the shape of the curve representing the depth distribution of $x$-ray generation.

If we call $\phi(\rho z)$ the intensity of $x$-rays generated in the specimen at depth $\rho z\left(\mathrm{~g} / \mathrm{cm}^{2}\right)$, the fraction of $\mathrm{x}$-rays being emitted after absorption in the specimen is equal to:

$$
f(\chi)=\int \phi(\rho z) \cdot \exp [(\mu / \rho) \cdot \csc \psi] \cdot \mathrm{d}(\rho z) / \int \phi(\rho z) \cdot \mathrm{d}(\rho z) .
$$

Here, $\rho$ is the density of the specimen, $z$ the linear depth, $\mu / \rho$ the $\mathrm{x}$-ray mass absorption coefficient, and $\psi$ the $x$-ray emergence angle. If these integrations were to be performed formally, the function yielding the values of $\phi(\rho z)$ had to be chosen in such a way that the Laplace transform of $\phi(\rho z)$ could be expressed algebraically. This restriction limited the choice of functions for absorption correction models. If the depth distribution function is, for instance, based on a Gaussian, as proposed by Andersen and Wittry [6] and later by Packwood and Brown [7], then an approximation to the error function would have to be used to calculate the integrals [8]. The equation proposed by Packwood for the emergent $x$-ray intensity is:

$$
\begin{aligned}
l_{\text {obs }}=C_{\mathrm{A}}(\sqrt{\pi} / 2 \alpha)\left(\gamma_{0} \cdot \exp (\chi / 2 \alpha)^{2} \cdot[1-\operatorname{erf}(\chi / 2 \alpha)]-\right. \\
\left.\left(\gamma_{0}-\phi_{0}\right) \cdot \exp [(\chi+\beta) / 2 \alpha]^{2} \cdot\{1-\operatorname{erf}[(\chi+\beta) / 2 \alpha]\}\right)
\end{aligned}
$$

where $\chi=(\mu / \rho) \cdot \csc \psi$ and $\alpha$ is a factor related to the width of the Gaussian. As the fraction $\chi / 2 \alpha$ rises above one, the error function rapidly approaches unity, so that the value between square brackets cannot always be calculated accurately with the approximation for the error function suggested by the author, even if the errors introduced in the binary calculation by the computer are insignificant. Hence, the calculation of intensity may fail at high values of $\chi$.

Even where no difficulties are observed with the application of the Laplace transformation, simple formulas for the $\phi(\rho z)$ curve may produce quite complicated expressions for the transform. For instance, the quadrilateral model of Scott and Love [9], in which the depth distribution is 
approximated by two straight lines, results in the following expression for the observed intensity, after absorption:

$$
\begin{aligned}
& f(\chi)=2 {\left[\left(\rho z_{\mathrm{r}}-\rho z_{\mathrm{m}}\right)\left(\rho z_{\mathrm{m}}+h \rho z_{\mathrm{r}}\right) \chi^{2}\right]^{-1} \cdot\left\{\left[-\exp \left(-\chi \rho z_{\mathrm{m}}\right)+h \exp \left(-\chi \rho z_{\mathrm{r}}\right)\right.\right.} \\
&\left.\left.+\chi\left(\rho z_{\mathrm{r}}-\rho z_{\mathrm{m}}\right)-h+1\right]+\left[\exp \left(-\chi \rho z_{\mathrm{m}}\right)\left(\rho z_{\mathrm{r}}-h \rho z_{\mathrm{r}}\right)+h \rho z_{\mathrm{r}}-\rho z_{\mathrm{r}}\right] / \rho z_{\mathrm{m}}\right\}
\end{aligned}
$$

in which $\rho z_{\mathrm{m}}$ and $\rho z_{\mathrm{r}}$ are the depth of maximum emission and the range, and $h$ is a function of the atomic number, of the operating potential, the critical excitation potential for the $x$-rays being measured, and of the backscatter coefficient.

The above mentioned limitations in the choice of expression for the depth distribution, and the failures that occasionally occur with various methods, can be avoided if $f(\chi)$ is obtained by numerical integration. It is not necessary to use procedures more complicated than adding rectangular strips of $\varphi(\rho z) \cdot \mathrm{d}(\rho z)$, and in most cases of homogeneous specimens, adding about 10 strips provides results which differ from those of a formal integration by no more than $0.1 \%$. It is therefore unnecessary to use more complex formulae for numerical integration. The time necessary for the numerical integration is often the same or shorter than that required for calculating the complicated equations that apply in the usual procedures; at any event, with modern computers, the time required for data collection usually exceeds that for the calculation.

Sometimes, the use of direct and explicit methods reveals unexpected limits in our present arsenal. Castaing wisely concluded that the key to accurate quantitative analysis was to compare intensities emitted by one element in the specimen with those from the same element in a specimen of known composition [1]. In the calculation of fluorescent effects, however, it is necessary to compare primary intensities emitted from different elements. The simplified fluorescence model of Reed et al [10] takes care of this problem, but it cannot be applied in its original form to some emissions, such as fluorescence caused by $M$ lines. The logical solution to the problem would be to calculate the fluorescent intensity from first principles, starting with the intensity emitted by the element causing fluorescence [11]. When this is attempted we find that we often do not know with the required precision the parameters that would allow us to compare primary intensities from different elements; hence, we observe unexpected errors. This problem has further implications for standardless analysis.

If there is one situation in which numerical integration shows its full power, it is in the calculation of fluorescence due to continuous radiation. J. Henoc, in his thesis, developed formulae for the formal calculation of this effect [12], which were later incorporated in a program for batch processing called COR [13]. The mathematical apparatus, and the resulting equations, are intimidating; furthermore, to allow for formal integration, Henoc had to accept simplifications (such as the growth of mass absorption coefficients with the third power of wavelength) which were less accurate than desirable. Fortunately, in most analytical situations, this effect is small, and is ordinarily neglected. However, it would be useful to include it, particularly in the emission of high energy $\mathrm{K}$ lines in matrices of low atomic number. The use of numerical integration [14] simplifies greatly the mathematical apparatus and allows the use of accurate models for mass absorption coefficients and other parameters. It is therefore easy to include the continuum fluorescence correction in the current procedures. This is not to say, however, that its inclusion in presently available procedures would necessarily improve the accuracy of the results, for two reasons: First, that it requires a good knowledge of the absolute intensity of continuous radiation from all types of targets including those of compound composition and over a wide range of energies, and secondly, that in the empirical adjustments underlying the procedures used at present, and which lack an explicit continuum fluorescence correction, the effects of this emission are probably contained implicitly, and adding an explicit correction may worsen the accuracy. 
The use of numerical integration in analytical procedures for stratified and coated specimens would also contribute to an easier understanding of the respective expressions, without in any way affecting the accuracy of the analytical results.

\section{References}

[1] CASTAING R., Doctoral Thesis, University of Paris (1951).

[2] Castaing R. and Descamps J., J. Phys. Radium 16 (1955) 304.

[3] Castaing R., Adv. Electr. Phys. 13 (1960) 317.

[4] Duncumb P. and REED S.J.B., Quantitative Electron Probe Microanalysis, NBS Special Publ. 298 (U.S. National Bureau of Standards, Washington D.C., 1968) p. 133.

[5] Philibert J., Proc. 3rd Int. Conf. on X-Ray Optics and Microanalysis, H.H. Pattee, V.E. Cosslett and A. Engström Eds. (Academic Press, New York, 1963) p. 379.

[6] ANDERSEN C.A. and WITTRY D.B., J. Phys. D. 1 (1968) 529.

[7] PACKWOOD R.H. and BROWN J.D., X-Ray Spectrometry 10 (1981) 138.

[8] PACKWOOD R.H., Electron Probe Quantitation, K.F.J. Heinrich and D.E. Newbury Eds. (Plenum Press, New York, 1981) p. 83.

[9] SCOTT V.D. and LOVE G., ibid., p. 19.

[10] READ S.J.B. and LONG J.V.P., Proc. 3rd Int. Conf. on X-Ray Optics and Microanalysis, H.H. Pattee, V.E. Cosslett and A. Engström, Eds. (Academic Press, New York, 1963) p. 317.

[11] HeinRICH K.F.J., Proc. 22d Annual Conf. of the Microbeam Analysis Society, Kona, Hawaii, 1987, R. Geiss Ed. (San Francisco Press, San Francisco, 1987) p. 23.

[12] HENOC J., Thesis, University of Paris (1962).

[13] HeNOC J., HEINRICH K.F.J. and MYKLEBUST R.L., NBS Tech. Note 769 (U.S. National Bureau of Standards, Washington D.C., 1973).

[14] HeInRICH K.F.J., Proc. 22d. Annual Conf. of the Microbeam Analysis Society, Kona, Hawaii, 1987, R. Geiss Ed. (San Francisco Press, San Francisco, 1987) p. 24. 